\title{
Hutchinson (Agavales) vs. Huber y Dahlgren (Asparagales): análisis moleculares sobre la fillogenia y evolución de la familia Agavaceae sensu Hutchinson dentro de las monocotiledóneas
}

\author{
LUIS E. EGUIARTE \\ Departamento de Ecología Evolutiva, Centro de Ecología, Universidad Nacional Autónoma de México, \\ Apartado Postal 70-275, C.P. 04510, D.F., México.
}

\begin{abstract}
Resumen: Para explorar diferentes ideas sobre la filogenia de las monocotiledóneas, y en particular sobre las Agavaceae sensu Hutchinson $(1934,1959)$ y su relación con grupos emparentados dentro de las Asparagales, se analizó la secuencia de ADN del gen del cloroplasto $r b c \mathrm{~L}$ para 134 especies (118 monocotiledóneas, empleando 16 dicotiledóneas paleohierbas como grupo externo). Se llevaron a cabo análisis de parsimonia utilizando métodos de distancia (neighbor-joining y UPGMA) y de máxima verosimilitud. Las filogenias muestran que Acorus calamus es la representante actual más cercana a las monocotiledóneas ancestrales. Los diferentes análisis identifican tres principales linajes evolutivos en las Agavaceae sensu Hutchinson: 1) El de las Agavaceae sensu stricto (Agave, Manfreda, Beschomeria, Hesperaloë y Yucca), Hosta (Funkiaceae) y Chlorophytum (Anthericaceae). 2) El grupo que incluye a las Nolinaceae (Nolina, Beaucarnea y Dasylirion), a Sansevieria (Dracaenaceae) y a Danae (Ruscaceae). 3) El formado por las Asphodelaceae (Aloë, Haworthia y Kniphofia) y Dianella (Phormiaceae). Estos linajes contienen a la mayoría de las Asparagales tal como fueron definidas por Huber (1969) y Dahlgren et al. (1985). También se estimó un reloj molecular para obtener las fechas de divergencia entre los grupos relacionados con las Agavaceae. Se calculó una tasa de $0.34 \times 10^{-9}$ substituciones nucleotídicas por sitio por año. Usando esta calibración se obtuvo un tiempo para el origen de las Agavaceae verdaderas (separación de Agavaceae-Hosta) de cerca de 14 millones de años, para la separación Agavaceae-Nolinaceae de hace unos 47 millones de años y para el origen de las Asparagales + Iridaceae + Cyanastraceae unos 84 millones de años. Sin embargo, estas estimaciones deben ser tomadas como preliminares.

Palabras clave: Agavaceae, Nolinaceae, filogenia, $r b c \mathrm{~L}$, monocotiledóneas, reloj molecular, evolución molecular.

Abstract: To explore different ideas in relation to the phylogeny of the monocotyledons, in particular about the Agavaceae sensu Hutchinson $(1934,1959)$ and their relationships with related groups of the Asparagales, the DNA sequence of the chlorplast gene $r b c \mathrm{~L}$ for 134 species (118 monocotyledons, 16 dicotyledon paleoherbs as an outgroup) was analyzed. Parsimony, distance (neighbor-joining and UPGMA) and maximum likelihood analyses were performed. The phylogenies show that Acorus calamus is the living plant more closely related to the ancestral monocotyledons. The different analyses identify three main evolutionary linages in the Agavaceae sensu Hutchinson: 1) One including the Agavaceae sensu stricto, (Agave, Manfreda, Beschorneria, Hesperaloë and Yucca) with Hosta (Funkiaceae) and Chlorophytum (Anthericaceae). 2) Other group that includes the Nolinaceae (Nolina, Beaucarnea and Dasylirion), Sansevieria (Dracaenaceae) and Danae (Ruscaceae). 3) A third group formed by the Asphodelaceae (Aloë, Haworthia and Kniphofia) and Dianella (Phormiaceae). These lineages include the majority of the Asparagales, as were defined by Huber (1969) and Dahlgren et al. (1985). A molecular clock was also estimated to obtain the divergence times among the groups related to the Agavaceae. A rate of $0.34 \times 10^{-9}$ nucleotide substitutions per site per year was obtained. Using this calibration, a date for the origin of the true Agavaceae (separation between Agavaceae-Hosta) was estimated to be about 14 million years, for the separation of the Agavaceae-Nolinaceae of about 47 million years and for the origin of the Asparagales + Iridaceae + Cyanastraceae of about 84 million years. Nevertheless, these estimates should be regarded as preliminary.
\end{abstract}

Key words: Agavaceae, Nolinaceae, phylogeny, $r b c \mathrm{~L}$, monocotyledons, molecular clock, molecular evolution.

\section{INTRODUCCIÓN}

Las monocotiledóneas actuales representan alrededor de 61,555 especies (2,744 géneros y 103 familias; Dahlgren et al., 1985), en comparación con las cerca de 176,075 especies de dicotiledóneas $(9,825$ géneros y 354 familias; Thorne, 1992). Sin embargo, a pesar de que las monocotiledóneas son tan abundantes, se sabe relativamente poco sobre su evolución (Eguiarte, en prensa). Por una parte, esto se debe a que las monocotiledóneas presentan altos grados de convergencia morfológica (Dahlgren et al., 1985). Por ejemplo los agaves y yucas, nolináceas, bromeliáceas, aloes, xantorroeas, dracenas, sansevierias, etc., crecen en lugares relativamente áridos similares en diferentes partes del mundo, y sus morfologías llegan a ser muy parecidas. Por otra, la mayoría de las monocotiledóneas son hierbas (más del 90\% de las especies), por lo que existen pocos restos fósiles.

En este artículo se analiza la ubicación de las Agavaceae sensu lato dentro de las monocotiledóneas. Las Agavaceae representan uno de los grupos que han generado más controversia y sobre los cuales se ha discutido mucho su posición y estatus sistemático. Los sistemas Englerianos (Bentham y Hooker, 1883; Pax y Hoffman, 1930; Krause, 1930) clasifican a las Agaváceas sensu lato en dos familias diferentes: 
Hutchinson, 1959

Dahlgren et al., 1985

Orden Agavales

Orden Asparagales

Familia Xanthorrhoeaceae

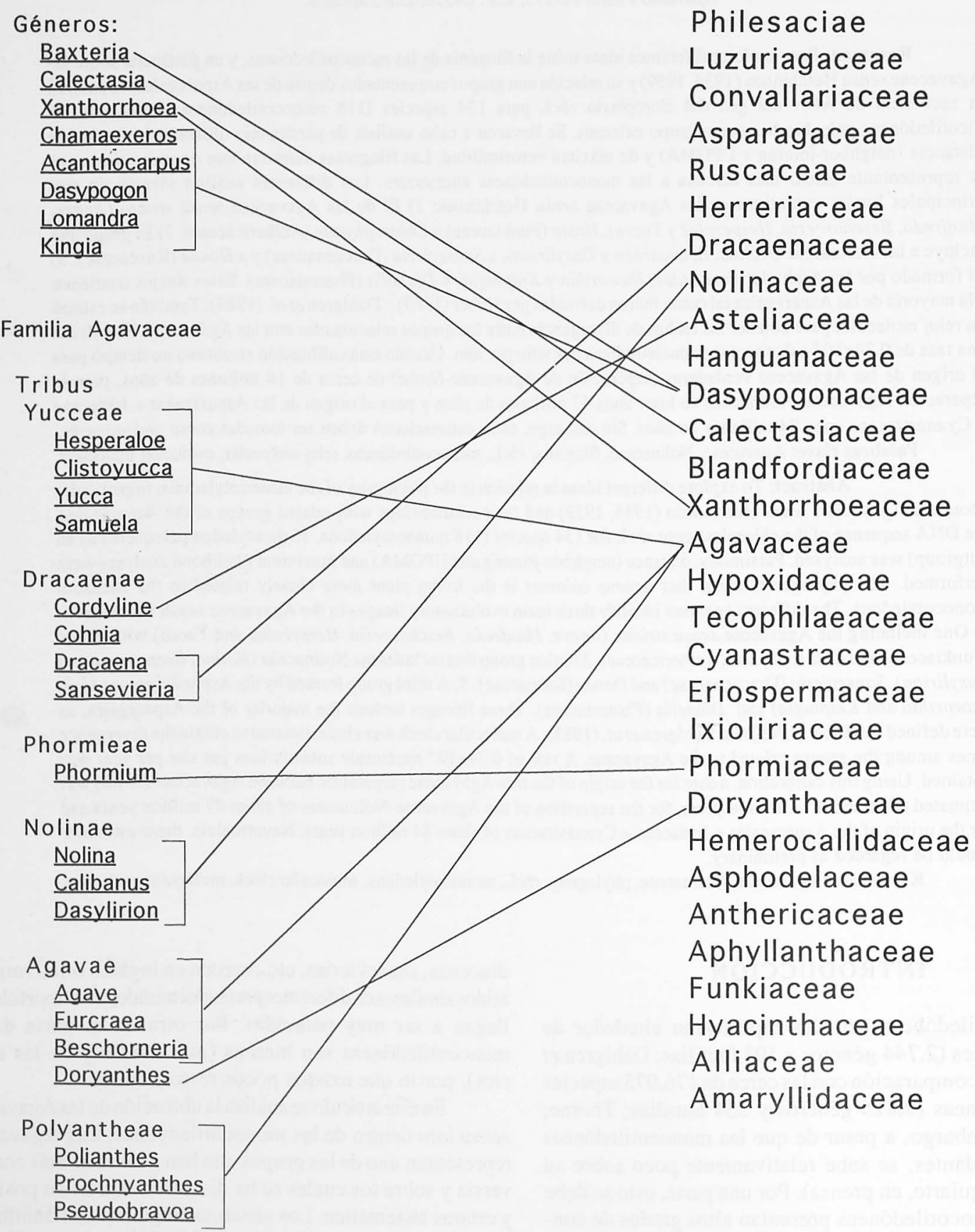

FIG. 1. Las Agavales de Hutchinson (1959) y las Asparagales de Dahlgren et al. (1985); se muestra las relaciones entre ambas propuestas. 
a) Amaryllidaceae, donde incluyen a Agave y géneros emparentados, por sus ovarios ínferos.

b) Liliaceae, que incluye a Yucca y géneros cercanos, por sus ovarios súperos.

Aunque la familia Agavaceae fue descrita en 1841 por Endlicher (incluyendo fundamentalmente a Furcraea y Agave), sus ideas tuvieron poco eco en la comunidad botánica, y no fue sino hasta 1934, año en que Hutchinson la vuelve a proponer (incluyendo 19 géneros, ver Fig. 1), cuando adquiere popularidad. Hutchinson (1959) también propuso el orden Agavales, formado por las familias Xanthorrhoeaceae y Agavaceae (Fig. 1). Las ideas de este autor han sido bastante populares entre los botánicos que trabajan en México (Reko, 1946; Gómez-Pompa et al., 1971; Gentry, 1972, etc.), sin embargo, en la comunidad botánica no existe consenso. Por ejemplo, la familia ha sido rechazada por McVaugh (1989), mientras que otros autores han tomado las ideas de Hutchinson con pocas o sin modificaciones (por ejemplo, Cronquist, 1981). Algunos botánicos han dividido a sus Agavaceae en otras familias (Takhtajan, 1980; Dahlgren et al., 1985). En concreto, Dahlgren et al.(1985), dividen a las Agavaceae sensu Hutchinson en seis familias dentro de su orden Asparagales, el cual tiene en total 30 familias (Fig. 1). El orden Asparagales fue propuesto por Huber (1969) basándose principalmente en la estructura y composición de la semilla (presencia de fitomelanina, estructura de la testa y el endospermo y el tamaño y forma del embrión), para incluir (en esa propuesta inicial) un total de 33 familias.

En este trabajo se analiza la posición y relaciones filogenéticas de difer entes géneros emparentados con la familia Agavaceae, tal como fue descrita originalmente por Hutchinson $(1934,1959)$ dentro de las monocotiledóneas, los análisis están basados en el gen $r b c \mathrm{~L}$ del cloroplasto. En términos generales, se utiliza la terminología del sistema de Dahlgren et al. (1985), excepto que se cambió la terminación «-iflorae» de los superórdenes, por «-anae» siguiendo las sugerencias de Thorne (1992) Chase et al. (1993) y Duvall et al. (1993b), que la consideran más apropiada.

\section{MATERIALES Y MÉTODOS}

Análisis filogenéticos. Para estos análisis se utilizó un total de 134 secuencias: 118 de monocotiledóneas, y como grupo externo (outgroup) 16 dicotiledóneas («paleohierbas», ver Chase et al., 1993), debido a que en estudios previos se había demostrado que ese grupo de dicotiledóneas era el más cercano a las monocotiledóneas (ver Hamby y Zimmer, 1992; Chase et al., 1993; Duvall et al., 1993a y b), y por lo tanto representaban el grupo externo adecuado. Los datos sobre estas secuencias (especie, familia, método de secuenciación, autor, etc.) se encuentran en los apéndices del Ann. Missouri Bot. Gard. 80: 767-785, en Duvall et al. (1993a) y en Eguiarte et al. (1994). Estas secuencias fueron analizadas con los métodos de UPGMA y de neighborjoining $(\mathrm{NJ})$, a partir de una matriz de distancias evolutivas calculada con el método de «dos parámetros» de Kimura (1981), utilizando el programa Phylip 3.5c para Macintosh de Felsenstein (1993) en una Quadra800. Asimismo, se realizó la búsqueda del conjunto de árboles más parsimoniosos mediante estrategias de búsqueda heurística NNI (nearestneighbor interchanges, ver Swofford y Olsen, 1990) con el programa PAUP 3.0s (Swofford, 1991). También, para un juego de nueve especies relacionadas con las Agavaceae sensu stricto, se llevó a cabo un análisis de máxima verosimilitud (maximum likelihood, Felsenstein, 1981) usando el programa DNAML del Phylip 3.5 de Felsenstein (1993).

Estimación de un reloj molecular. Para tener una idea de los tiempos en que surgieron las Agavaceas sensu stricto y cuándo se separaron de los otros grupos con los cuales están supuestamente relacionadas, se aproximó un reloj molecular. Para esto se estimó la tasa de substitución promedio a partir de la comparación de la $K$ (número de substituciones estimadas con el método de Kimura (1981) de dos parámetros para pares de especies) promedio entre nueve paleohierbas y 118 monocotiledóneas, considerando que ambos grupos se separaron hace unos 150 millones de años (ver Dahlgren $e t$ al., 1985 y Eguiarte, en prensa). Mediante estos valores se ajustó un eje de tiempo en una reconstrucción filogenética empleando el algoritmo UPGMA para 28 especies cercanas a las Asparagales sensu Dahlgren et al. (1985), usando también la distancia de dos parámetros de Kimura, todo calculado con el programa Phylip, versión, 3.5 (Felsenstein, 1993). También se aproximó un reloj molecular utilizando varias estimaciones contrastantes de las tasas de substitución por sitio por año en cloroplasto para las angiospermas (Zurawski y Clegg, 1987, Wilson et al., 1990; Clegg et al., 1991; Eguiarte, en prensa).

\section{RESULTADOS}

134 especies. El primer análisis de reconstrucción filogenética, obtenido por el método de UPGI 1 A, resulta muy distorsionado por la gran heterogeneidad en las tasas de substitución en el gen rbcL (Gaut et al., 1992; Bousquets et al., 1992; Eguiarte et al., 1994) al unir en la base a los grupos con tasas de substitución más altas (por ejemplo, algunas dicotiledóneas con los pastos, etc.); debido a esto, se consideró que no valía la pena mostrar dicha reconstrucción. Sin embargo, el otro método de distancia, el NJ, es más resistente a las heterogeneidades (Hillis et al., 1994), y es congruente con árboles equivalentes obtenidos por parsimonia y máxima verosimilitud para menos especies, empleando la secuencia de $r b c L$ por Duvall et al. (1993a y b). La reconstrucción filogenética obtenida con el método de NJ se muestra en la Fig. 2 y uno de 7,200 árboles igualmente parsimoniosos obtenidos se ilustra en la Fig. 3. A partir de ambos árboles se puede señalar que los principales grupos de monocotiledóneas son:

1. Acorus calamus, que aparece en la base, y como hemos señalado (Duvall et al., 1993a; Eguiarte, en prensa) parece ser el pariente vivo más cercano a las monocotiledóneas ancestrales. 


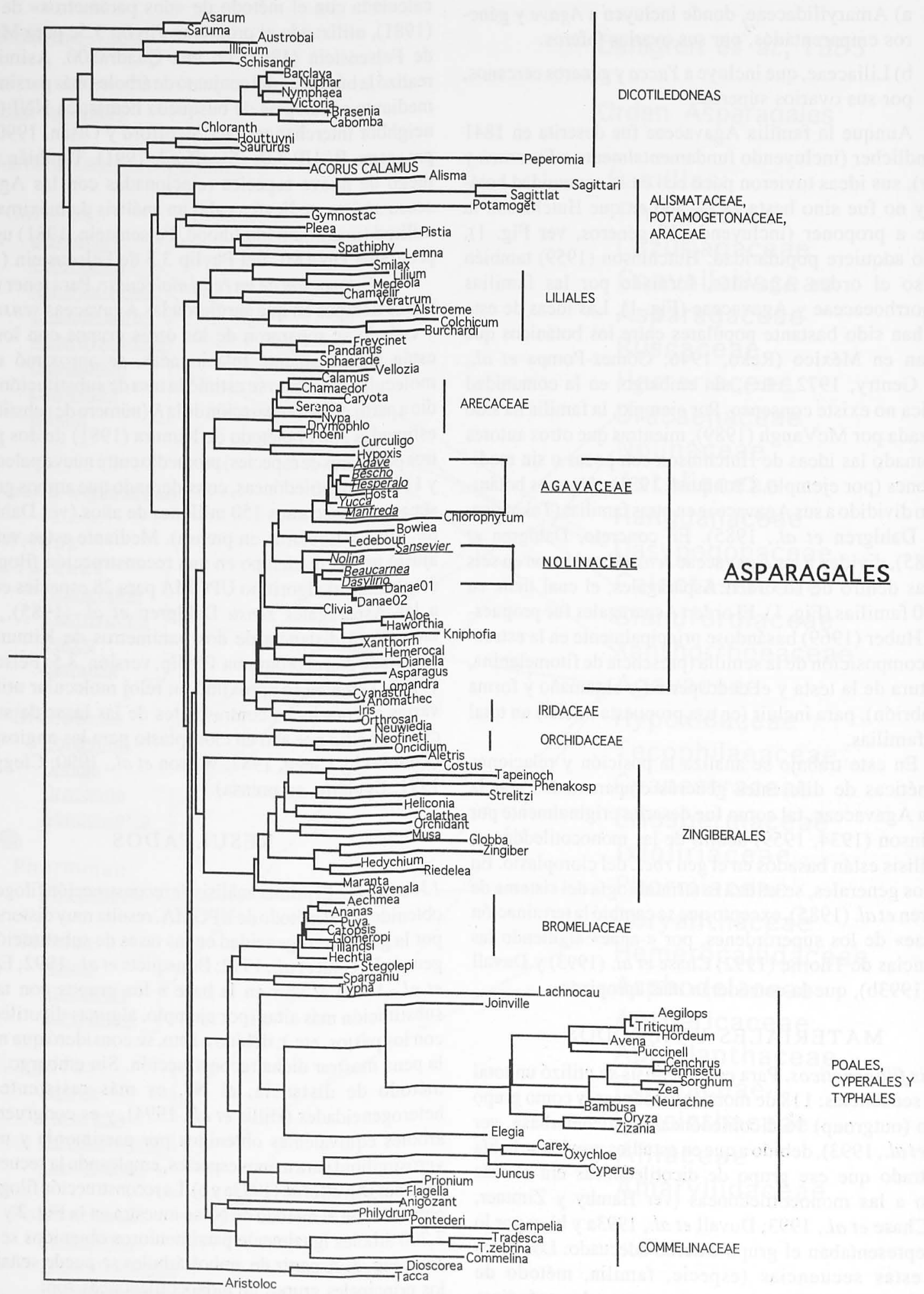

FIG. 2. Análisis de distancia por el método de neighbor-joining, empleando las distancias obtenidas con el modelo de dos parámetros de Kimura (1981) para 118 monocotiledóneas y 16 especies de paleohierbas como grupo externo. 


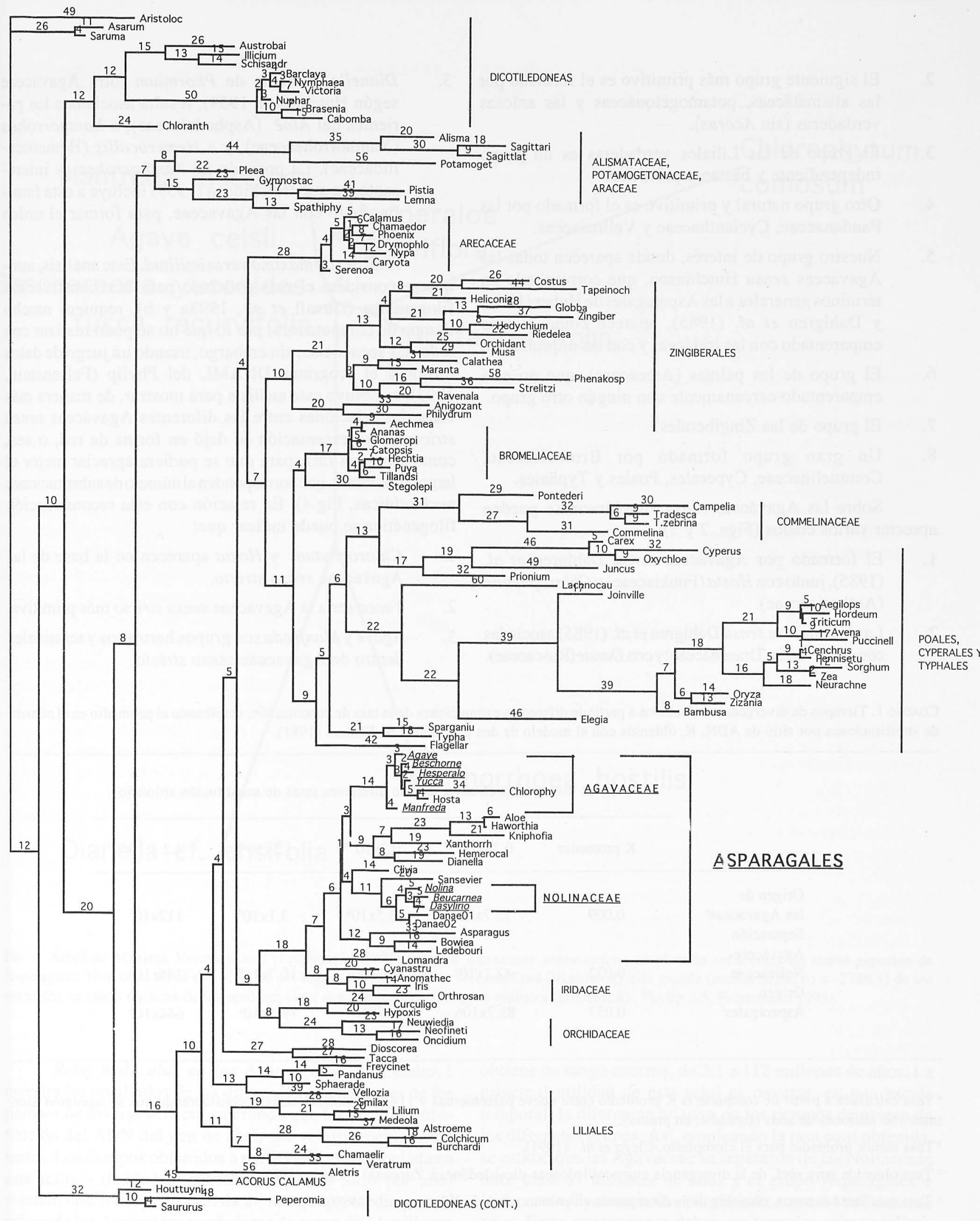

FIG. 3. Árbol tomado al azar de los 7,200 igualmente parsimoniosos (largo = 4,190 pasos, Índice de Consistencia = 0.288, Índice de Retención $=0.634$ ) para 118 especies de monocotiledóneas y 16 especies de paleohierbas como grupo externo. Los números indican el número de pasos (substituciones). 
2. El siguiente grupo más primitivo es el formado por las alismatáceas, potamogetonáceas y las aráceas verdaderas (sin Acorus).

3. El grupo de las Liliales verdaderas es-un grupo independiente y bastante primitivo.

4. Otro grupo natural y primitivo es el formado por las Pandanaceae, Cyclanthaceae y Velloziaceae.

5. Nuestro grupo de interés, donde aparecen todas las Agavaceas sensu Hutchinson, que corresponde en términos generales a las Asparagales de Huber (1969) y Dahlgren et al. (1985), aparece como el más emparentado con las iridáceas y con las orquidáceas.

6. El grupo de las palmas (Arecaceae), que no está emparentado cercanamente con ningún otro grupo.

7. El grupo de las Zingiberales.

8. Un gran grupo formado por Bromeliaceae, Commelinaceae, Cyperales, Poales y Typhales.

Sobre las Agaváceas sensu Hutchinson se pueden apreciar varios clados (Figs. 2 y 3 ):

1. El formado por Agavaceae sensu Dahlgren et al. (1985), junto con Hosta (Funkiaceae) y Chlorophytum (Anthericaceae).

2. Las Nolinaceae sensu Dahlgren et al. (1985) asociadas con Sansevieria (Dracenaceae) y con Danae (Ruscaceae).
3. Dianella, pariente de Phormium (otra Agavaceae según Hutchinson, 1959), resulta asociada a los parientes del Alö̈ (Asphodelaceae), a Xanthorrohea (Xanthorroheaceae), y a Hemerocallis (Hemerocallidaceae). La posición de Xanthorrohea es interesante, ya que Hutchinson (1959) incluye a esta familia, junto con las Agavaceae, para formar el orden Agavales.

Análisis de máxima verosimilitud. Este análisis, aunque se considera el más poderoso para la reconstrucción filogenética (Duvall et al., 1993a y b), requiere mucho tiempo de computadora, por lo que no se pudo realizar con todas las secuencias; sin embargo, usando un juego de datos menor y el programa DNAML del Phylip (Felsenstein, 1993), se obtuvo este análisis para mostrar, de manera más clara, las relaciones entre las diferentes Agaváceas sensu stricto (la representación se dejó en forma de red, o sea, como árbol sin raíz, para que se pudiera apreciar mejor el largo de las ramas, que corresponden al número de substituciones nucleotídicas, Fig.4). En relación con esta reconstrucción filogenética se puede indicar que:

1. Chlorophytum y Hosta aparecen en la base de las Agavaceae sensu stricto.

2. Yucca sería la Agavaceae sensu stricto más primitiva.

3. Agave y Manfreda son grupos hermanos y terminales dentro de Agavaceae sensu stricto.

CuAdro 1. Tiempos de divergencia calculados a partir de diferentes estimaciones de la tasa de substitución, empleando el promedio en el número de substituciones por sitio de ADN, K, obtenida con el modelo de dos parámetros de Kimura (1981).

Tiempo de divergencia en años, usando diferentes tasas de substitución sitio/año)

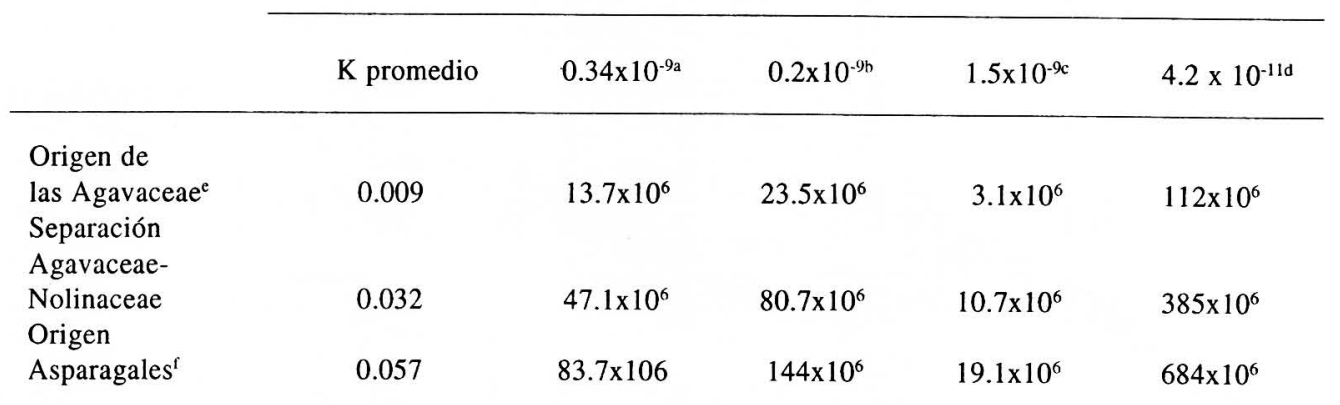

\footnotetext{
a Tasa calculada a partir de comparar la $\mathrm{K}$ promedio entre nueve paleohierbas y 118 monocotiledóneas, y considerando que se separaron hace unos 150 millones de años (Eguiarte, en prensa).

${ }^{\mathrm{h}}$ Tasa menor promedio para el cloroplasto, Clegg et al. (1991).

c Tasa obtenida para $r b c \mathrm{~L}$ de la divergencia monocotiledóneas-dicotiledóneas, Zurawski y Clegg (1987).

'Tasa más lenta extrema, obtenida de la divergencia en palmas en $r b c \mathrm{~L}$, Wilson et al. (1990).

' Separación Hosta-Agavaceae sensu stricto.

${ }^{\prime}$ Curculigo e Hypoxis al resto de las Asparagales + Iridaceae + Cyanastraceae.
} 


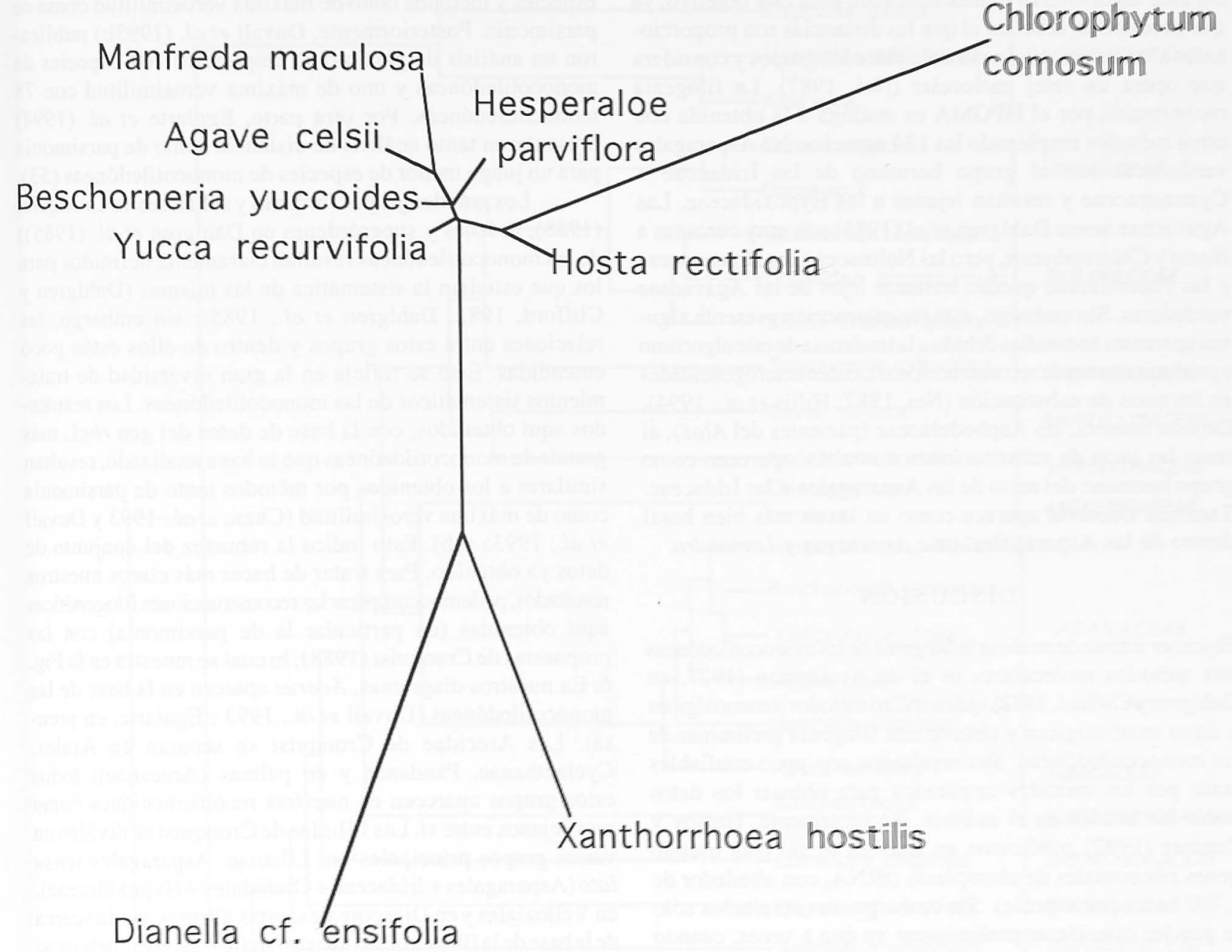

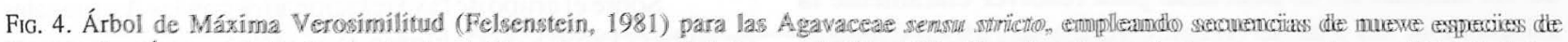

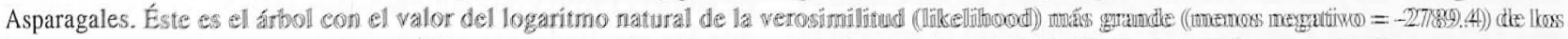

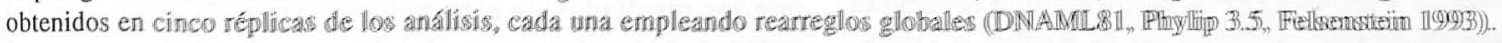

Reloj molecular en las Asparagales. El Cuadro 1 muestra los resultados de las diferentes estimaciones de los tiempos de divergencia considerando que las tasas de substitución del ADN del gen de rbeL son relativamente constantes. Los tiempos obtenidos a partir de la tasa calculada en este trabajo $\left(0.34 \times 10^{-4}\right.$ substituciones por sitio por afio) resultan intermedios, lo cuall da un tiempo promedio para el origen de las Agavacede verdaderas de cerca de 14 millones de años. Sin embarigo, contino se puede observar en el cuadro 1, usando las diferentes tasas teportadas en la literatura se

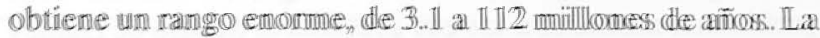

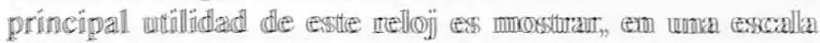

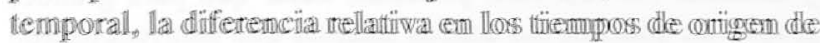

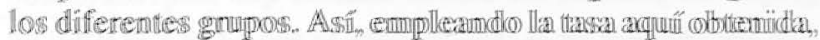

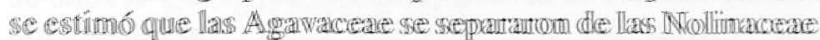
hace unos 47 milllomes de affios: y ell grtupo A.spratragalless +

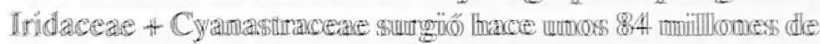

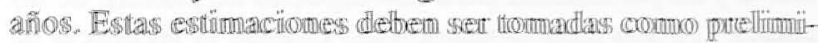

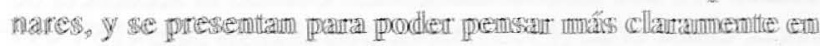

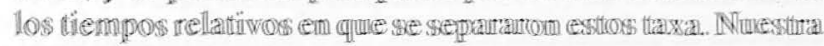


estimaciôn del tiempo de separación entre las monocotiledóneas y las dicotiledóneas se incorporó en la reconstrucción obtenida mediante el algoritmo UPGMA para 28 especies (Fig. 5). Este algoritmo es el más adecuado para este objetivo, ya que produce un árbol en el que las distancias son proporcionales a las diferencias promedio entre los grupos y considera que opera un reloj molecular (Nei, 1987). La filogenia reconstruida por el UPGMA es análoga a la obtenida con otros métodos empleando las 134 especies: las Asparagales verdaderas son el grupo hermano de las Iridaceae + Cyanastraceae y resultan lejanas a las Hypoxidaceae. Las Agavaceae sensu Dahlgren et al. (1985) son muy cercanas a Hosta y Chlorophytum, pero las Nolinaceae, las Dracenaceae y las Phormiaceae quedan bastante lejos de las Agavaceae verdaderas. Sin embargo, esta reconstrucción presenta algunas aparentes anomalias debido a la tendencia de este algoritmo a producirerrores de reconstrucción siexisten heterogeneidades en las tasas de substitución (Nei, 1987; Hillis et al., 1994). De esta manera, las Asphodelaceae (parientes del Aloë), al tener las tasas de substituciones mas altas, aparecen como grupo hermano del resto de las Asparagales + las Iridaceae. También Dianella aparece como un taxon mâs bien basal dentro de las Asparagales, ente Asparagus y Lomandra.

\section{DISCUSION}

Ell primer intento de analizar la fillogenia de las monocotiledóneas con métodos moleculares es el de Amkerman (1927, en Dahlgren y Clifford, 1982), quien utilizó métodos inmunológicos y datos morfológicos y obtuvo una fĩlogenia preliminar de las monocotiledóneas. Sus resultados son poco confiables tanto por los métodos empleados para obtener los datos como los usados en el análisis. Recientemente, Hamby y Zimmer (1992) publicaron un análisis de 60 taxa usando genes ribosomales de cloroplasto ( $\mathrm{rRNA}$, con alrededor de 1,700 bases por especie). Sim embargo, sus resultados sólo se puedem considerat prelimimares ya que a veces, cuando incluxem una mueva secuencia en su analisis, cambia la topología de todo ell árbol; por eso se considera que la cantidad de información en las secuencias y/o que ell número de las mismas mo es adecuadio para resolver claramente la fillogentia de las plantas (Hamby y Zirmmer, 1992; Chase et (4l.., 1993). Martion y Dowd (1991), empleando la secuencia de ADN para la unidad pequeña de la RuBisCo (rbes, inferida a partir de la secuencia de amimoácidos) analizaron 335 especies de plantas. Sim embargo, su análisis tiene problemas similares a los que presenta ell trabajo de Hamby y Zimmer (1992). Asú, podemos decir que para grandes grupos de plantas, los úmicos análisis que han functionado som los basadios em la rbel. (Chase et al., 1993 y ell resto de ese volumen dell Amm. Miss. Bot. Gard.). Eim este trabajo presentamos análisis a partir de matrices de distancia evolutiva y de parsimomia para. 134 especies de plantas, de las cuales 118 som monocotiledtóneas. Usando la secuencia de rbect. Chase et al. (1993) publicarom análisis para 475 y 499 especies de plantas con semillas, pero incluyverom sólo de 91 a 93 especies de monocotiledóneas, y emplearon exclusivamente análisis de parsimonia. Duvall et al.(1993a) presentaron análisis de la filogenia de monocotiledóneas usando 27 especies y métodos tanto de máxima verosimilitud como de parsimonia. Posteriormente, Duvall et al. (1993b) publicaron un análisis de parsimonia empleando 104 especies de monocotiledóneas y uno de máxima verosimilitud con 78 monocotiledóneas. Por otra parte, Eguiarte et al. (1994) presentaron tanto análisis de distancia como de parsimonia para un juego menor de especies de monocotiledóneas (53).

Los grandes grupos (órdenes y subclases en Cronquist (1988), órdenes y superórdenes en Dahlgren et al. (1985)) de las monocotiledóneas resultan claramente definidos para los que estudian la sistemática de las mismas (Dahlgren y Clifford, 1982; Dahlgren et al., 1985); sin embargo, las relaciones entre estos grupos y dentro de ellos están poco entendidas. Esto se refleja en la gran diversidad de tratamientos sistemáticos de las monocotiledóneas. Los resultados aquí obtenidos, con la base de datos del gen $r b c L$ más grande de monocotildeóneas que se haya analizado, resultan similares a los obtenidos por métodos tanto de parsimonia como de máxima verosimilitud (Chase et al., 1993 y Duvall et al., 1993a y b). Esto indica la robustez del conjunto de datos ya obtenido. Para tratar de hacer más claros nuestros resultados, podemos comparar las reconstrucciones filogenéticas aquî obtenidas (en particular la de parsimonia) con las propuestas de Cronquist (1988), lo cual se muestra en la Fig. 6. En nuestros diagramas, Acorus aparece en la base de las monocotiledóneas (Duvall et al., 1993 ; Eguiarte, en prensa). Las Arecidae de Cronquist se separan en Arales, Cyclanthanae, Pandanae y en palmas (Arecanae); todos estos grupos aparecen en nuestras reconstrucciones como muy lejanos entre sí. Las Liliidae de Cronquist se dividen en varios grupos principales: en Lilianae, Asparagales sensu lato (Asparagales + Iridaceae + Orchidales + Hypoxidaceae), en Velloziales y en Dioscoreales; estas últimas quedan cerca de la base de la filogenia. En nuestro análisis, las Bromeliaceae están separadas de las Zingiberanae. Las Commelinidae de Cronquist se parten en Commelinaceae, Poales, Cyperales y Thyphales.

Sobre el grupo de taxa relacionados con las Asparagales de Huber (1969) y de Dahlgren et al. (1985), se puede indicar que los resultados de este trabajo apoyan la existencia del orden Asparagales sensu Dalghren et ah.(1985), aunque el ajuste no es perfecto, y sugiere la remoción de las Hypoxidaceae de las Asparagales y la inclusión de las Iridaceae. Este análisis muestra claramente la posición de las Agavaceae y Agavales semsu Hutchinson dentro de las momocotiledóneas y se puede ver que constituyen un grupo relativamente compacto, al quedar todas dentro de las Asparagales de Huber (1969) y de Dahlgren et ab. (1985), pero en realidad constituyen un grupo heterogéneo, parafilético. Es notable la existencia de cuando menos dos grandes grupos que corresponden, gnasso modo, con las divisiones en nivel familia propuestas por Dahlgren et chl (1995): las Agavaceae sensu striction, y las Nolinaceae semsu Dahlgren et al. (1985). 


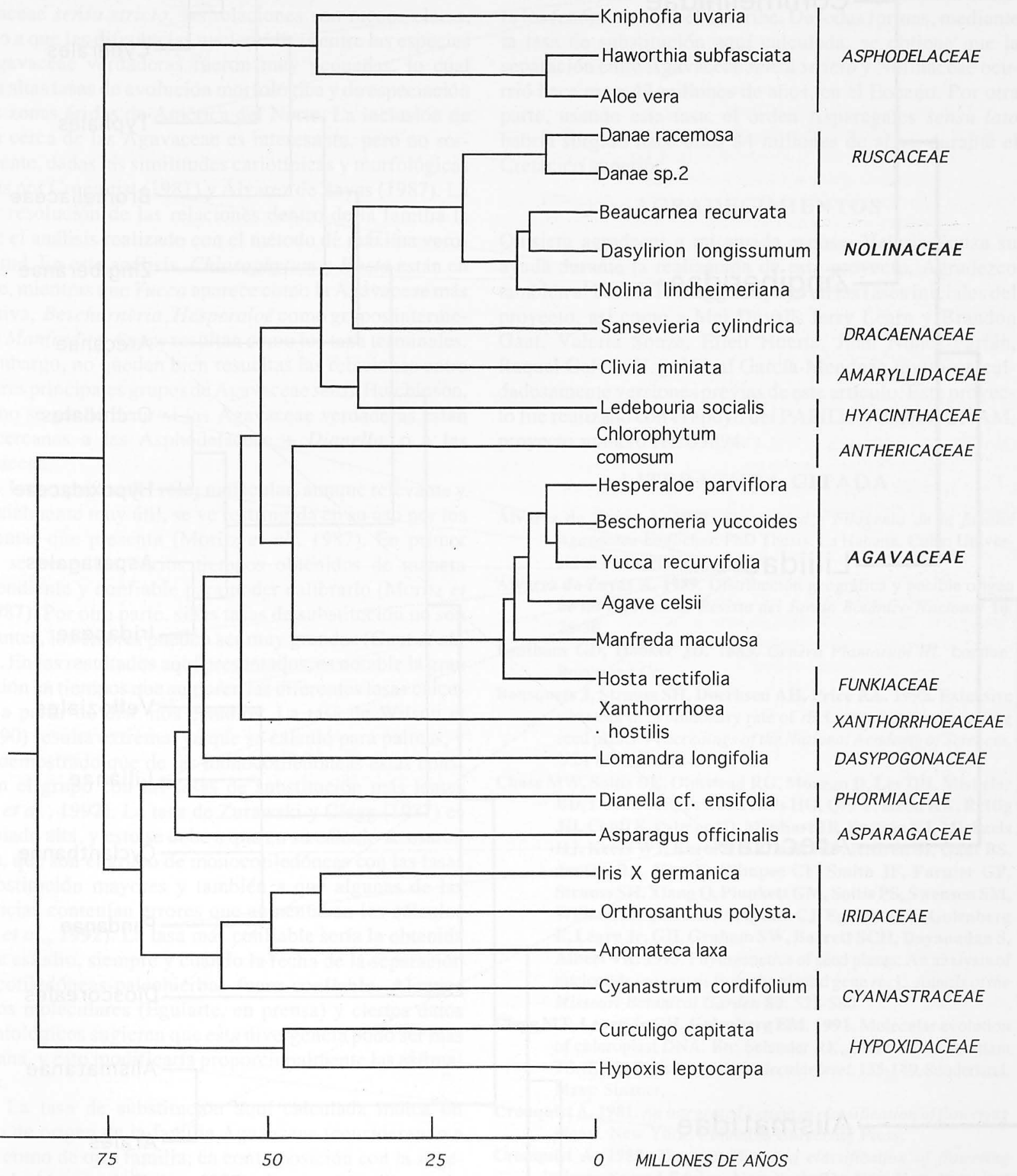

FIG. 5. Árbol de distancia calculado por el algoritmo UPGMA para 28 especies de Asparagales y grupos cercanos, empleando las distancias obtenidas con el modelo de dos parámetros de Kimura (1981). El largo de las ramas indica la distancia evolutiva. También se incluye una aproximación a un reloj molecular empleando una tasa de substitución de $0.34 \times 10^{-9}$ substituciones por sitio por año (Cuadro 1 ). 


\section{Cronquist, 1988}

rbcL

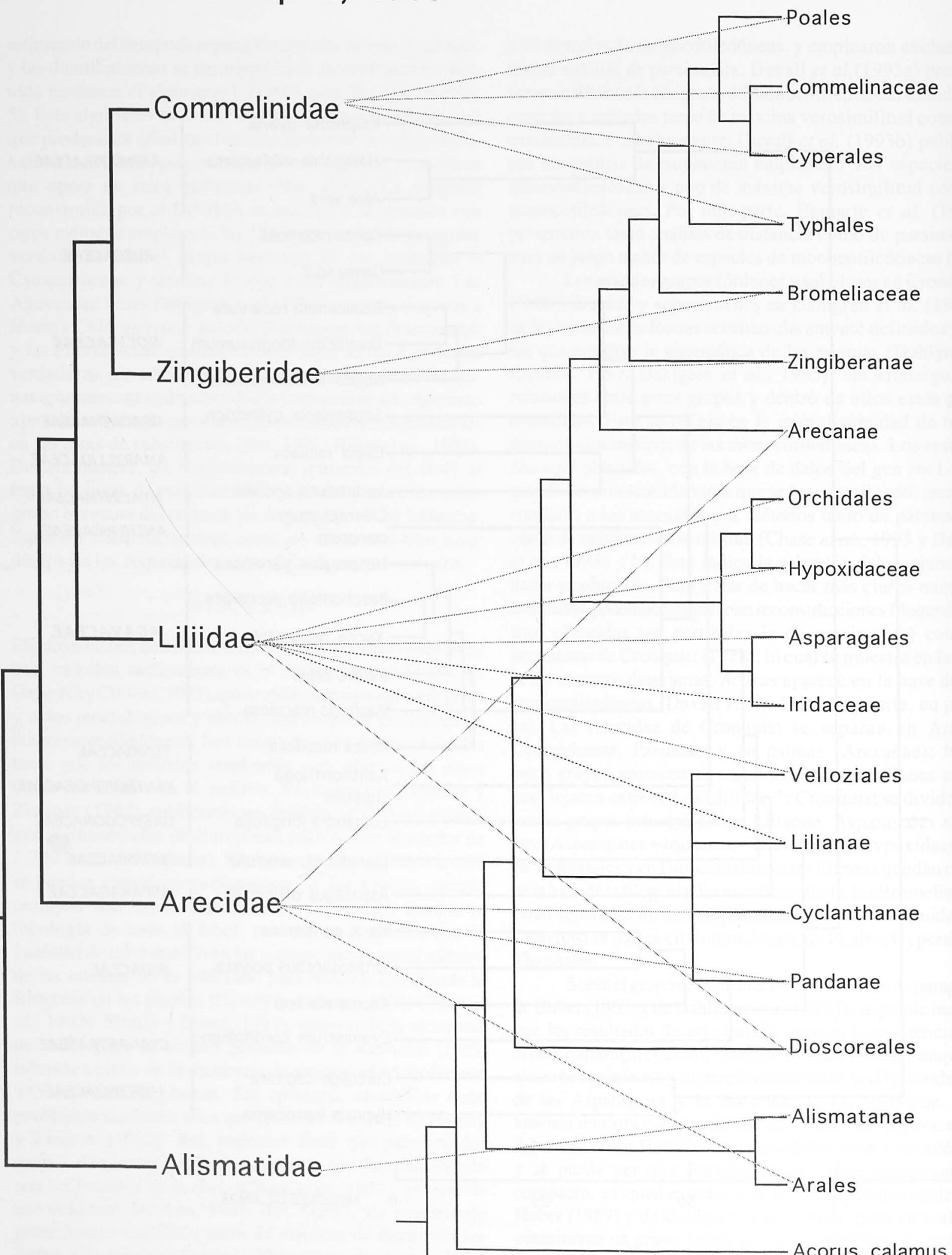

Acorus calamus

Paleohierbas (Dico)

Fig. 6. Relaciones entre la propuesta de evolución para las monocotiledóneas de Cronquist (1988), y los resultados presentados aquí obtenidos a partir de la secuencia $r b c L$, en particular nuestros árboles de parsimonia (Fig. 3). 
Las Agavaceae sensu stricto resultan cercanas a la hierba pantropical Chlorophytum (Anthericaceae), y a la hierba Hosta (Funkiaceae) nativa de China y Japón. Dentro de las Agavaceae sensu stricto, las relaciones son menos claras, debido a que las diferencias nucleotídicas entre las especies de Agavaceae verdaderas fueron muy pequeñas, lo cual indica altas tasas de evolución morfológica y de especiación en las zonas áridas de América del Norte. La inclusión de Hosta cerca de las Agavaceae es interesante, pero no sorprendente, dadas las similitudes cariotípicas y morfológicas citadas por Cronquist (1981) y Álvarez de Zayas (1987). La mejor resolución de las relaciones dentro de la familia la ofrece el análisis realizado con el método de máxima verosimilitud. En este análisis, Chlorophytum y Hosta están en la base, mientras que Yucca aparece como la Agavaceae más primitiva, Beschorneria, Hesperaloë como grupos intermedios y Manfreda y Agave resultan como los taxa terminales. Sin embargo, no quedan bien resueltas las relaciones entre estos tres principales grupos de Agavaceae sensu Hutchinson, y así no se puede decir si las Agavaceae verdaderas están más cercanas a las Asphodelaceae + Dianella, o a las Nolinaceae.

La propuesta del reloj molecular, aunque relevante y potencialmente muy útil, se ve restringida en su uso por los problemas que presenta (Moritz et al., 1987). En primer lugar, se necesitan varios tiempos obtenidos de manera independiente y confiable para poder calibrarlo (Moritz et al., 1987). Por otra parte, si las tasas de substitución no son constantes, los errores pueden ser muy grandes (Gaut et al., 1992). En los resultados aquí presentados, es notable la gran variación en tiempos que sugieren las diferentes tasas calculadas a partir de dist ntos estudios. La tasa de Wilson et al.(1990) resulta extrema, ya que se calculó para palmas, y se ha demostrado que de las monocotiledóneas éstas constituyen el grupo con las tasas de substitución más lentas (Gaut et al., 1992). La tasa de Zurawski y Clegg (1987) es demasiado alta, y esto se debe a que en su cálculo se usaron pastos, que son el grupo de monocotiledóneas con las tasas de substitución mayores y también a que algunas de las secuencias contenían errores que aumentaban los cálculos (Gaut et al., 1992). La tasa más confiable sería la obtenida en este estudio, siempre y cuando la fecha de la separación monocotiledóneas-paleohierbas fuera confiable. Algunos trabajos moleculares (Eguiarte, en prensa) y ciertos datos paleontológicos sugieren que esta divergencia pudo ser más temprana, y esto modificaría proporcionalmente las estimaciones.

La tasa de substitución aquí calculada indica un tiempo de origen de la familia Agavaceae (considerando a Hosta como de otra familia, en contraposición con la sugerencia de Álvarez de Zayas, 1987), de unos 14 millones de años. Para las Agavaceae el registro fósil es prácticamente nulo (Álvarez de Zayas, 1987 y 1989); sin embargo, esta fecha parece adecuada, debido a que corresponde, cuando menos en orden de magnitud, a la señalada por Álvarez de Zayas (1989). Él sugiere, a partir de consideraciones bioge ográficas, que la familia se originó en el Eoceno (hace 37-57 millones de años). Sin embargo, fechas de origen de la familia más recientes podrían explicar de la misma manera los fenómenos que él describe. De todas formas, mediante la tasa de substitución aquí calculada, se obtiene que la separación entre Agavaceae sensu stricto y Nolinaceae ocurrió hace unos 47 millones de años, en el Eoceno. Por otra parte, usando esta tasa, el orden Asparagales sensu lato habría surgido hace unos 84 millones de años, durante el Cretácico superior.

\section{AGRADECIMIENTOS}

Quisiera agradecer a mi amada esposa, Valeria Souza su ayuda durante la realización de este proyecto. Agradezco también al Dr. M. T. Clegg su apoyo en las fases iniciales del proyecto, así como a Mel Duvall, Jerry Learn y Brandon Gaut. Valeria Souza, Elleli Huerta, Juan Nuñez-Farfán, Raquel Galván V. y Abisaí García-Mendoza revisaron cuidadosamente versiones previas de este artículo. Este proyecto fue realizado con el apoyo del PAPIIT, DGAPA, UNAM, proyecto número IN205894.

\section{LITERATURA CITADA}

Álvarez de Zayas A. 1987. Sistemática y Filogenia de la familia Agavaceae Endlicher. PhD Thesis. La Habana, Cuba: Universidad de La Habana.

Álvarez de Zayas A. 1989. Distribución geográfica y posible origen de las Agavaceae. Revista del Jardín Botánico Nacional 10: 25-36.

Bentham GD, Hooker JD. 1883. Genera Plantarum III. London: Reeve and Co.

Bousquets J, Strauss SH, Doerksen AH, Price RA. 1992. Extensive variation in evolutionary rate of $r b c \mathrm{~L}$ gene sequences among seed plants. Proccedings of the National Academy of Sciences, USA 89: 7844-7848.

Chase MW, Soltis DE, Olmstead RG, Morgan D, Les DH, Misheler BD, Duvall MR, Price R, Hills HG, Qiu Y, Kron KA, Rettig JH, Conti E, Palmer JD, Manhart JR, Systma KJ, Michaels HJ, Kress WJ, Karol KG, Clark WD, Hedrén M, Gaut BS, Jansen RK, Kim K, Wimpee CF, Smith JF, Furnier GF, Strauss SH, Xiang Q, Plunkett GM, Soltis PS, Swensen SM, Williams SE, Gadek PA, Quinn CJ, Eguiarte LE, Golenberg E, Learn Jr. GH, Graham SW, Barrett SCH, Dayanadan S, Albert VA. 1993. Phylogenetics of seed plants: An analysis of nucleotide sequences from the plastid gene $r b c \mathrm{~L}$. Annals of the Missouri Botanical Garden 80: 528-580.

Clegg MT, Learn Jr GH, Golenberg EM. 1991. Molecular evolution of chloroplast DNA. En: Selander RK, Clark AG, e Whittam TS.(Eds.).Evolution at the molecularlevel. 135-149. Sunderland, Mass: Sinauer.

Cronquist A. 1981. An integrated system of classification of flowering plants. New York: Columbia University Press.

Cronquist A. 1988. The evolution and classification of flowering plants. Second Edition. New York: The New York Botanical Garden.

Dahlgren RMT, Clifford HT.1982. The Monocotyledons: A comparative study. London: Academic Press.

Dahlgren RMT, Clifford HT, Yeo PF. 1985. The families of the monocotyledons. Structure, Evolution and Taxonomy. Berlin: Springer-Verlag. 
Duvall MR, Learn Jr GH, Eguiarte LE, Clegg MT. 1993a. Phylogenetic analysis of $r b c L$ sequences identifies Acorus calamus as the primal extant monocotyledon. Proccedings of the National Academy of Sciences, USA 90: 4641-4644.

Duvall MR, Clegg MT, Chase MW, Clark WD, Kress JW, Hills HG, Eguiarte LE, Smith JF, Gaut BS, Zimmer A, Learn, Jr. GH. 1993b. Phylogenetic hypothesis for the Monocotyledons constructed from $r b c \mathrm{~L}$ sequence data. Annals of the Missouri Botanical Garden 80: 607-619.

Eguiarte LE (en prensa) Filogenia y evolución de las monocotiledóneas. En: Nuñez-Farfán J, Zamora JC. (Eds.) Tópicos de biología evolutiva II. México, D.F.: Facultad de Ciencias, UNAM.

Eguiarte LE, Duvall MR, Learn, Jr GH, Clegg MT.1994. The systematic status of the Agavaceae and Nolinaceae and related Asparagales in the Monocotyledons: An analysis based on the rbcL gene sequence. Boletín de la Sociedad Botánica de México 54: 35-56.

Endlicher IL. 1841. Enchiridion botanicum.. Lepzing: Engelmann.

Felsenstein J. 1981. Evolutionary sequences from DNA sequences: A maximum likelihood approach. Journal of Molecular Evolution 17: 368-376.

Felsenstein J. 1993. Phylip (Phylogeny Inference Package). Seattle: University of Washington.

Gaut BS, Muse SV, Clark WD, Clegg MT. 1992. Relative rates of nucleotide substitution at the $r b c \mathrm{~L}$ locus of monocotyledonous plants. Journal of Molecular Evolution 35: 292-303.

Gentry HS. 1972. The Agave family in Sonora. Washington: Agriculture Handbook No. 399. United States Department of Agriculture.

Gomez-Pompa A, Villalobos-Pietrini R, Chimal A. 1971: Studies in the Agavaceae. I. Chromosome morphology and number of seven species. Madroño 21: 208-211.

Hamby RK, Zimmer EA. 1992. Ribosomal RNA as a phylogenetic tool in plant systematics. En: Soltis PS, Soltis DE, Doyle JJ. (Eds.). Molecular systematics of Plants. 50-91. New York: Chapman Hall.

Hillis DM, Huelsenteck JP, Cunningham CW. 1994. Application and accuracy of molecular phylogenies. Science 264: 671-677.

Huber H. 1969. Die Samenmekmale und Verwandstchftverhältnisse der Liliifloren. Mitt Bot Staatssamml Muenchen 8: 219-538.

Hutchinson J. 1934. The families of flowering plants. Vol. II. Monocotyledons. London: The Macmillan Co.
Hutchinson J. 1959. The families of flowering plants. Vol. II. Monocotyledons. 2a. ed. Oxford: Clarendon Press.

Kimura M. 1981. Estimation of evolutionary distances between homologous nucleotide sequences. Proccedings of the National Academy of Sciences, USA 78: 454-458.

Krause K. 1930. Liliaceae. En: Engler A. (Ed). Pflanzenreich, 15a. 351-362.Leipzig: Engelmann.

Martin PG, Dowd JM. 1991. Studies of angiosperm phylogenies using protein sequences. Annals of the Missouri Botanical Garden 78: 296-337.

Mcvaugh R. 1989. Flora Novo-Galiciana. A descriptive account of the vascular plants of Western Mexico. Vol. 15: Bromeliaceae to Dioscoreaceae. Ann Arbor: The University of Michigan Herbarium. Ann Arbor.

Moritz C, Dowling TE, Brown WD. 1987. Evolution of animal mitochondrial DNA: Relevance for population biology and systematics. Annual Review of Ecology and Systematics 18: 269-292.

Nei M. 1987. Molecular Evolutionary Genetics. New York: Columbia University Press.

Pax F,Hoffman K. 1930. Amaryllidaceae. En: Engler A. ed. Pflanzenreich, 15a. 391-430. Leipzig: Engelmann.

Reko BP. 1946. Los géneros fanerogámicos mexicanos. Boletín de la Sociedad Botánica de México 4: 19-40.

Swofford DL. 1991. PAUP 3.Os: Phylogenetic Analysis Using Parsimony. User's Manual. Champaign, Illinois: Natural History Survey.

Swofford DL, Olsen GJ. 1990. Phylogeny reconstruction. En: Hillis DM, Moritz C. (Eds.) Molecular Systematics. 411-501. Sunderland, Mass: Sinauer.

Takhtajan AL. 1980. Outline of the classification of flowering plants (Magnoliophyta). Botanical Review 46: 225-359.

Thorne ReF.1992. An updated classification of flowering plants. Aliso 13: 365-389.

Wilson MA, Gaut B, Clegg MT. 1990. Chloroplast DNA evolves slowly in the palm family. Molecular Biology and Evolution 7: 303-314.

Zurawski G, Clegg MT. 1987. Evolution of higher-plant chloroplast DNA-encoded genes: implications for structure-function and phylogenetic studies. Annual Review of Plant Physiology 38: 391-418. 